\title{
Pathogenesis of Tuberculosis: Interaction of Mycobacterium tuberculosis with Macrophages
}

\author{
KATHLEEN A. MCDONOUGH, ${ }^{1,2} \dagger$ YVONNE KRESS, ${ }^{2}$ AND BARRY R. BLOOM ${ }^{1,2 *}$ \\ Howard Hughes Medical Institute ${ }^{1 *}$ and Albert Einstein College of Medicine, ${ }^{2}$ \\ 1300 Morris Park Avenue, Bronx, New York 10461
}

Received 27 January 1992/Accepted 12 April 1993

\begin{abstract}
Central to understanding the pathogenesis of tuberculosis is the interaction between the pathogen and mononuclear phagocytes. A key question about that interaction is whether Mycobacterium tuberculosis exerts an effect on phagolysosome fusion. We have reexamined the dynamics of phagolysosome fusion and its effect on intracellular bacterial replication in $M$. tuberculosis-infected macrophages by performing an extensive study at the electron microscopic level. Thoria-labelled murine and human macrophages were infected with a virulent (H37Rv) or avirulent (H37Ra) strain of M. tuberculosis or with Mycobacterium bovis BCG vaccine for times ranging from 2 h to 7 days. In all cases, by 2 h postinfection, approximately $85 \%$ of the bacteria clearly resided in fused vacuoles. However, at 4 days postinfection, fusion levels for viable H37Rv and H37Ra were reduced by half, whereas the fusion profiles of BCG and of heat-killed H37Rv and H37Ra were unchanged. $A$ comparison of the numbers of bacteria per fused and nonfused vacuoles suggests both a net transfer of bacteria out of fused vacuoles and preferential bacterial multiplication in nonfused vacuoles. H37Rv and H37Ra appeared to bud from the phagolysosomes into tightly apposed membrane vesicles that did not fuse with secondary lysosomes. In some cases, no such membrane was seen and the bacteria appeared to be free in the cytoplasm. Only viable H37Rv showed a significant increase in bacterial counts during the course of infection. Thus, both of the attenuated strains we examined difiered from the virulent strain $\mathbf{H 3 7 R v}$ in their abilities to replicate successfully within macrophages, but each diverged from H37Rv at a difierent point in the process. Viable tubercle bacilli H37Rv and H37Ra had the capacity to escape from fused vesicles as the infection progressed; BCG did not. After extrusion from the phagolysosome, H37Rv, but not H37Ra, was able to multiply. These results suggest a novel mechanism by which virulent $M$. tuberculosis eludes the microbicidal mechanisms of macrophages by escaping from fused phagolysosomes into nonfused vesicles or the cytoplasm.
\end{abstract}

Mycobacterium tuberculosis, the etiologic agent of tuberculosis, is responsible for more deaths each year than any other single pathogen (21). Once thought to be nearing eradication in developed countries, tuberculosis is undergoing a dramatic resurgence in the United States and throughout the world. The emergence of significant levels of multiple-drug-resistant $M$. tuberculosis isolates further complicates treatment and control, underscoring the need to develop new strategies for prevention and treatment of tuberculosis beyond currently available antimicrobial therapies (4).

The rational design of a more effective vaccine and better chemotherapeutic agents against tuberculosis requires a better understanding of the pathogenesis of infection, particularly during the early stages, about which little is known. Aerosol transmission is the predominant route of infection, whereby droplet nuclei containing one to three bacilli (particle size, $<5 \mu \mathrm{m})$ gain access to alveoli $(26,27,39)$. Here the bacteria are engulfed by alveolar macrophages, which presumably are equipped with multiple microbicidal mechanisms, including phagolysosome fusion and a respiratory burst, to rid the host of infecting microorganisms $(34,38)$. To establish infection successfully, the tubercle bacillus must survive its encounter with the alveolar macrophages and ultimately gain access to the lymphatics or the bloodstream $(6,7,25)$. To gain insight into these events and to clarify

\footnotetext{
* Corresponding author.

† Present address: Axelrod Institute for Public Health, Albany, NY 12201-0509.
}

important early steps in the pathogenesis of tuberculosis infection, we have performed an extensive study of several parameters of the mycobacterium-macrophage interaction.

It is generally believed that killing and growth inhibition of intracellular pathogens within cells of the mononuclear phagocyte lineage are dependent on phagolysosome fusion (20). Strategies employed by other intracellular parasites for surviving phagolysosome fusion include inhibition of the fusion process (44), preferential use of uptake pathways that do not lead to phagolysosomal fusion (17), resistance to the lysosomal contents (16), inactivation of lysosomal enzymes $(12,18)$, modification of the phagolysosome to facilitate survival and replication of the parasite $(35,36)$, and escape into the cytoplasm $(24,29)$. It is unclear which of these strategies, if any, pathogenic mycobacteria use to survive within the host.

Previous attempts to address this question have yielded confusing results. Pioneering studies by Armstrong and Hart (1) indicated that virulent tubercle bacilli can inhibit phagolysosome fusion and that the fusion pattern of macrophages infected with virulent tubercle bacilli is affected by the presence or absence of serum components at the time of infection. In this context, it has been shown that $M$. tuberculosis can enter macrophages through complement receptors CR1 and CR3 (30). These findings suggest that the route of uptake may influence the course of events following ingestion of tubercle bacilli by macrophages. Therefore, mycobacteria may survive their interaction with host phagocytic cells by utilizing uptake pathways that do not result in phagolysosomal fusion or that fail to signal an appropriate respiratory burst or other cytocidal mechanisms $(20,42)$. 
However, Armstrong and Hart (2) later demonstrated that while phagolysosome fusion could be achieved in mouse macrophages by opsonizing $M$. tuberculosis with antibodies before uptake, the pathogen nevertheless remained fully able to survive under these conditions. Furthermore, earlier studies provided evidence that phagolysosome fusion may in fact be stimulatory to the growth of tubercle bacilli within macrophages (5), an observation that has never been satisfactorily explained by later findings. Clearly, the significance of phagolysosome fusion to the intracellular survival of $M$. tuberculosis remains problematic. We have therefore reexamined phagolysosome fusion in $M$. tuberculosis infection of macrophages in vitro. Specifically, we have explored differences in the intracellular interactions with macrophages among three mycobacterial strains, including the virulent $M$. tuberculosis strain H37Rv, the avirulent strain H37Ra derived from $\mathrm{H} 37 \mathrm{Rv}$, and the attenuated Mycobacterium bovis BCG vaccine strain originally derived from a bovine tubercle bacillus isolate, to identify bacterial phenotypes that may be involved in the pathogenesis of $M$. tuberculosis infection.

\section{MATERIALS AND METHODS}

Bacterial strains and growth conditions. $M$. tuberculosis H37Ra (ATCC 25177) and H37Rv (ATCC 25618) were reconstituted from lyophilized stocks with sterile distilled water and grown for 2 weeks in standing culture in liquid $7 \mathrm{H} 9$ medium (Difco) supplemented with $0.5 \%$ glycerol, $10 \%$ oleic acid-albumin-dextrose complex (Difco), and $0.25 \%$ Tween 80. Cultures were then diluted with an equal volume of growth medium and frozen at $-70^{\circ} \mathrm{C}$ in $1-\mathrm{ml}$ aliquots. Before use, aliquots were thawed, diluted into liquid growth medium, and grown for 5 to 7 days at $37^{\circ} \mathrm{C}$ without shaking. M. bovis BCG (Pasteur strain, Trudeau Institute) cultures were started from frozen aliquots and passaged weekly at 1:50 up to the ninth pass. Bacteria were usually used after 5 to 7 days of growth, at a density of approximately $1 \times 10^{7}$ to $5 \times 10^{7}$ bacteria per ml. Standing cultures grown in this way form a fine silt-like precipitate that can be resuspended with agitation or vortexing, generating uniform suspensions of small clumps containing 1 to 10 bacteria. Mycobacterium smegmatis $\mathrm{mc}^{2} 155$ was diluted from a frozen stock into liquid medium (without oleic acid) and used after 1 day of growth. Viable counts were determined after appropriate dilutions and plating on solid $7 \mathrm{H} 10$ bacteriologic medium (Difco) supplemented with $0.5 \%$ glycerol, $10 \%$ oleic acidalbumin-dextrose complex, and $0.01 \%$ cycloheximide. Colonies were counted after 2 to 3 days for $M$. smegmatis and 2 to 3 weeks for BCG and M. tuberculosis.

Tissue culture. The mouse macrophage cell line J774.16 (9) was maintained in antibiotic-free Dulbecco's modified Eagle's medium (GIBCO) containing $20 \%$ fetal bovine serum (Hyclone Laboratories, Inc., Logan, Utah), 5\% NCTC 109 (GIBCO), $1 \%$ nonessential amino acids (Gibco), and $1 \%$ glutamine. Cells were seeded at a density of approximately 5 $\times 10^{5}$ cells per ml 18 to 24 h before use. Lab-tek Permanox eight-chamber microscopy slides (Nunc, Inc., Naperville, Ill.) were used for electron microscopy studies, with $200 \mu \mathrm{l}$ of medium added per well.

Isolation of primary human monocytes. Blood from nonimmune donors was drawn into heparinized tubes and diluted 3:5 with sterile phosphate-buffered saline (PBS) at $37^{\circ} \mathrm{C}$ before being layered onto a Ficoll-Paque (Pharmacia) gradient. Gradients were centrifuged at $1,400 \mathrm{rpm}$ for $30 \mathrm{~min}$. The buffy coat was removed and washed twice in sterile saline and once in serum-free medium. Cells were resuspended in
Iscove's medium (GIBCO) supplemented with $10 \%$ heatinactivated normal human $\mathrm{AB}$ serum, $1 \%$ glutamine, and $1 \%$ sodium pyruvate. The cell suspension $(200 \mu \mathrm{l})$ was seeded in each well of Lab-tek Permanox eight-chamber microscope slides. Cells were agitated several times over an 18-h period and then washed to remove nonadherent cells. This technique resulted in 50 to $70 \%$ confluent monolayers of adherent cells.

Thoria labelling. Lysosomes were labelled with thoria $(2 \%$ solids; Polysciences, Warrington, Pa.) as described by Straley and Harmon (37) with the following modifications. Thoria was diluted 1:10 into tissue culture medium and then vortexed to dissolve the precipitate that forms. This suspension was then sterilized by being filtered through a $0.2-\mu \mathrm{m}$ low-protein-binding filter (Gelman) attached to a syringe. Tissue culture medium was removed from the cell monolayers and replaced with the medium containing thoria for $3 \mathrm{~h}$ at $37^{\circ} \mathrm{C}$ with $5 \% \mathrm{CO}_{2}$. The labelling medium was replaced with fresh maintenance medium after the cells were washed twice with sterile PBS or serum-free medium. Cells were incubated for an additional 12 to $15 \mathrm{~h}$ before being infected to ensure uptake of residual thoria and its delivery to lysosomes.

Infection of monolayers. Bacterial cultures were diluted at least 1:20 directly into fresh tissue culture medium to achieve the desired multiplicity of infection. This inoculating medium was used to replace the maintenance medium for periods of 1.5 to $6 \mathrm{~h}$ at $37^{\circ} \mathrm{C}$ with $5 \% \mathrm{CO}_{2}$. After this infection period, monolayers were washed twice with PBS or serumfree medium and returned to the incubator with fresh medium for the duration of the experiment. Typically, multiplicities of infection in the range of 10 bacilli per cell were used, resulting in approximately 20 to $30 \%$ of the cells becoming infected with 8 to 12 bacilli per cell. For experiments of 7 or more days, the multiplicity of infection was reduced up to 10 -fold. The cell culture medium was replaced with fresh medium daily unless otherwise specified.

Fixation for electron microscopy. At appropriate time points, the monolayers were washed twice with serum-free medium and fixed for $45 \mathrm{~min}$ on ice in a solution of $2 \%$ glutaraldehyde and $2 \%$ osmium tetroxide $(1: 1)$ in $0.1 \mathrm{M}$ cacodylate buffer ( $\mathrm{pH}$ 7.3). The fixed monolayers were washed twice (10 min each wash) with $0.1 \mathrm{M}$ cacodylate buffer, rinsed in distilled water, and left standing overnight in $4 \%$ paraformaldehyde with $1 \%$ glutaraldehyde. After several rinses, the monolayers were placed in $1 \%$ tannic acid for $1 \mathrm{~h}$ and then dehydrated in ascending ethanol to a mixture of $100 \%$ ethanol-Spurr's resin, diluted $3: 1,1: 1$, and $1: 3$ for $2 \mathrm{~h}$ each time. The monolayers were then infiltrated with pure Spurr's resin over 2 days, embedded by placing inverted BEEM capsules over the cells, and cured at $65^{\circ} \mathrm{C}$ for 2 days. Thin sections were stained in $3 \%$ uranyl acetate for $20 \mathrm{~min}$ and lead citrate for 2 min before they were examined in a Siemens 102 electron microscope.

Electron microscopy analysis. For each sample, at least four grids containing sections cut from different regions throughout the sample were analyzed. Routinely, data were collected from 25 cells on each of four grids. The number of bacteria in each cell was recorded, resulting in counts from a total of 100 cells per sample. From these data, the percentage of cells infected, the number of bacteria per 100 cells, and the average number of bacteria per infected cell were calculated.

To assay phagolysosome fusion, at least four random fields containing bacteria from each grid were photographed at a magnification of $\times 18,000$ to $\times 30,000$, resulting in a total of approximately 17 prints ( 8 by 10 in. [ca. 20 by $25 \mathrm{~cm}$ ]) per 


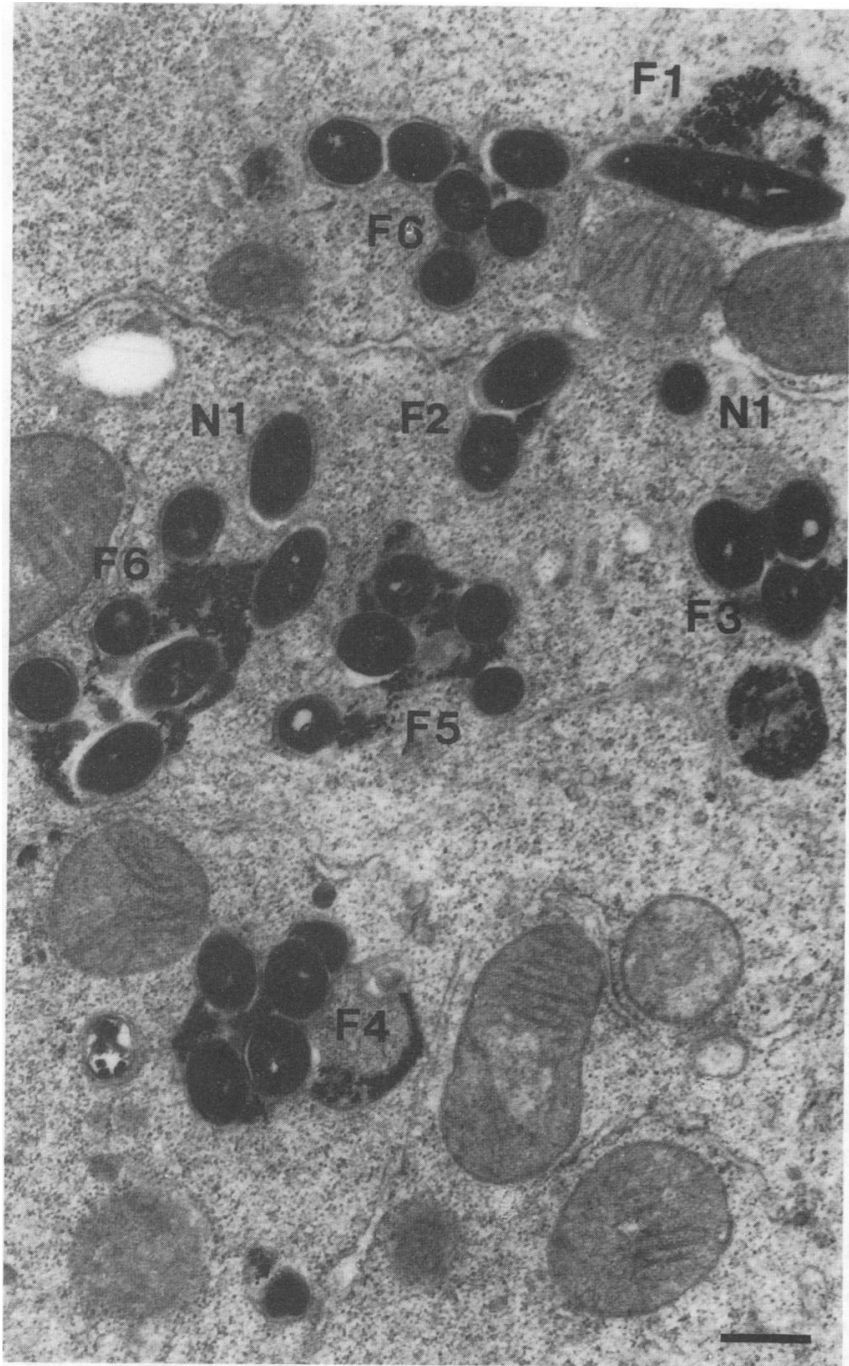

FIG. 1. Typical field of BCG-infected J774.16 macrophages shown at one-fourth the magnification used to score phagolysosome fusion in this study. F, fused vacuole; $N$, nonfused vacuole. Numbers represent the number of bacteria in each vacuole. Note the presence of thoria granules in fused but not in nonfused vesicles. Bar, $0.5 \mu \mathrm{m}$.

sample. By using these prints, each vacuole was scored for the number of bacteria it contained and the presence or absence of thoria particles as illustrated in Fig. 1. When thoria was visible within a vacuole containing bacteria, phagolysosome fusion was inferred to have taken place, and that vacuole was scored as fused. Vacuoles with no thoria present were considered nonfused, and in questionable cases the vacuole was scored as indeterminate. Data were compiled on (i) the number of vacuoles in each of the above categories, (ii) the total number of bacteria falling into each of the three groups, and (iii) the average number of bacteria per vacuole in each category. The percent fusion for either vacuoles or bacteria was calculated as the number fused divided by the total counted.

\section{RESULTS}

Phagolysosome fusion after infection with virulent and avirulent $M$. tuberculosis strains. Previous studies addressing

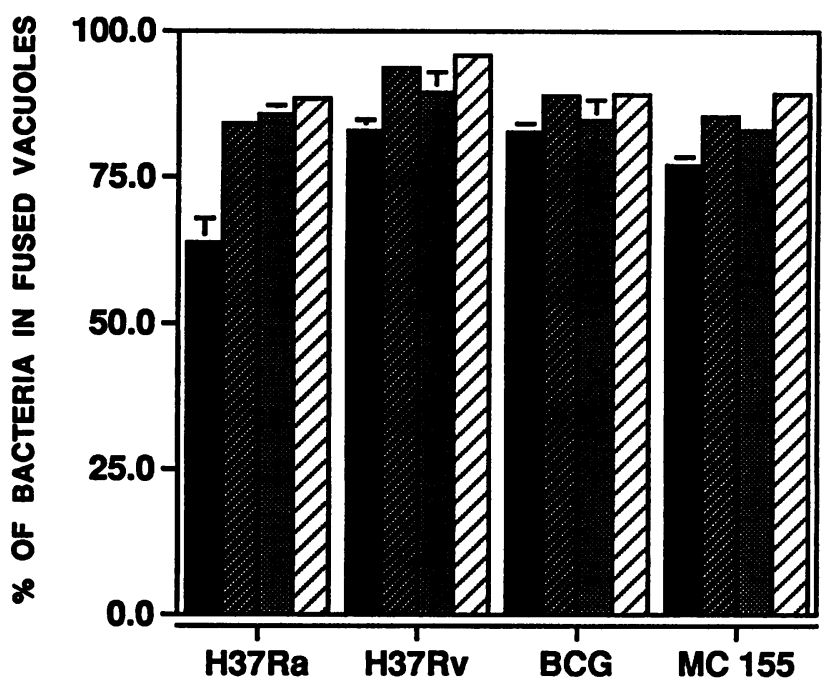

FIG. 2. Phagolysosome fusion levels assessed at 2 and $6 \mathrm{~h}$ postinfection in J774.16 cells infected with various strains of viable and heat-killed mycobacteria. Error bars represent standard errors of three independent experiments with viable H37Ra, H37Rv, and BCG bacteria and two experiments for $M$. smegmatis $\mathrm{mc}^{2} 155$. Results shown for heat-killed bacteria were derived from a single experiment. Symbols: $\square$, live bacteria at $2 \mathrm{~h} ; \mathbf{Z}$, dead bacteria at $2 \mathrm{~h} ; \square$, live bacteria at $6 \mathrm{~h} ; \ddot{\mathbb{B}}$, dead bacteria at $6 \mathrm{~h}$.

the question of phagolysosome fusion of tubercle bacilli in macrophages assessed the degree of fusion beginning 1 or 2 days postinfection $(1,2)$. It is now known that lysosomes fuse with endosomes containing virus particles within $30 \mathrm{~min}$ of their internalization (31). While less is known about the kinetics of phagolysosome fusion after bacterial uptake by macrophages, it is reasonable to assume that the timing of lysosome fusion with phagosomes containing bacteria is similar to that which is observed with other internalized particles. To delineate the early events involving phagolysosome fusion with mycobacteria, we examined the number of mycobacteria in fused and unfused phagosomes at 2 and 6 $h$ postinfection.

Cells of the murine macrophage-like cell line J774.16 (9) were prelabeled with thoria solids, a marker for secondary lysosomes, for 12 to $16 \mathrm{~h}$ before they were infected with various virulent and avirulent mycobacterial strains, including both viable and heat-killed organisms. In all experiments, approximately $85 \%$ of the bacteria resided in phagolysosomes at $6 \mathrm{~h}$ postinfection, regardless of their viability at the time of infection (Fig. 2). With the exception of viable H37Ra bacteria, maximum fusion levels were achieved as early as $2 \mathrm{~h}$ postinfection. Viable, but not heat-killed, H37Ra showed a lower level of phagolysosomal fusion upon entry, such that at $2 \mathrm{~h}$ postinfection the level of fusion was only $75 \%$ of the maximum reached by $6 \mathrm{~h}$.

Phagolysosome fusion in human monocytes. Since transformed murine macrophage-like cell lines may not always reflect the properties of primary cells, and some interspecies differences have been reported between mouse and human macrophages with respect to their ability to kill and degrade mycobacteria (28), we extended these experiments to primary human monocytes. Peripheral blood monocytes were isolated from normal, tuberculin-negative donors, cultured for 2 days, and infected with H37Ra, H37Rv, and BCG. As shown in Fig. 3, results of phagolysosome fusion in primary 


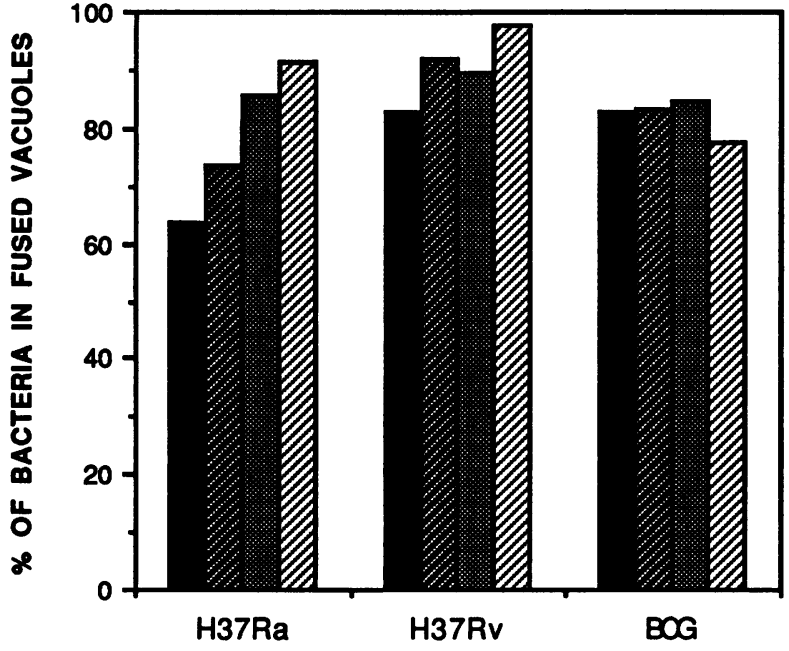

FIG. 3. Comparison of phagolysosome fusion levels in $\mathbf{J 7 7 4 . 1 6}$ cells with those in human monocytes from tuberculin-negative donors infected with various strains of mycobacteria. Fusion was assessed in a single experiment at 2 and $6 \mathrm{~h}$ postinfection. Symbols:

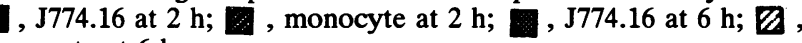
monocyte at $6 \mathrm{~h}$.

human monocytes were remarkably similar to those observed in J774.16 macrophages.

The fusion pattern of $M$. tuberculosis reverses with time. We performed several experiments, with daily time points up to 7 days, to examine the long-term interactions between both viable and heat-killed mycobacteria and macrophages. In the case of heat-killed bacteria of any strain, little change was observed in the monolayers. Levels of phagolysosome fusion increased slightly over time, gradually approaching $100 \%$. Bacterial degradation was apparent in these samples but appeared to be a slow and inefficient process, since organisms could be found as late as 14 days postinfection. In contrast to our findings with heat-killed bacteria, the levels of phagolysosome fusion in J774.16 cells infected with viable H37Rv and H37Ra bacteria began to decrease between 1 and 2 days postinfection. After 3 to 4 days, the fusion pattern had completely reversed, with fewer than $50 \%$ of the bacteria now residing in fused vacuoles. In contrast, phagolysosome fusion patterns in cells infected with viable BCG remained constant over the same time periods (Fig. 4). Occasional degraded bacteria were observed in these cells, but these represented a minority of organisms.

Changes in bacterial and vacuolar morphology occur with time. Additional morphological changes, illustrated in Fig. 5, were also noted over the course of these long-term infections. These differences included changes in both the appearance of the bacteria and the nature of the vacuoles in which they resided. At early times postinfection and after in vitro culture in bacteriologic medium, the morphologies of all three bacterial strains were similar. All had well-defined internal structures in a dense cytoplasm, surrounded by several layers of membrane and cell wall. After intracellular growth (or extracellular growth in tissue culture medium), the phenotype of H37Rv takes on a bull's-eye-like appearance (Fig. 5e). The centers of these organisms contain a darkly staining solid core, possibly composed of nucleic acid, surrounded by a lightly staining region which contains a network of filaments that extends out of the central core. Surrounding this region is a zone of dense cytoplasm,

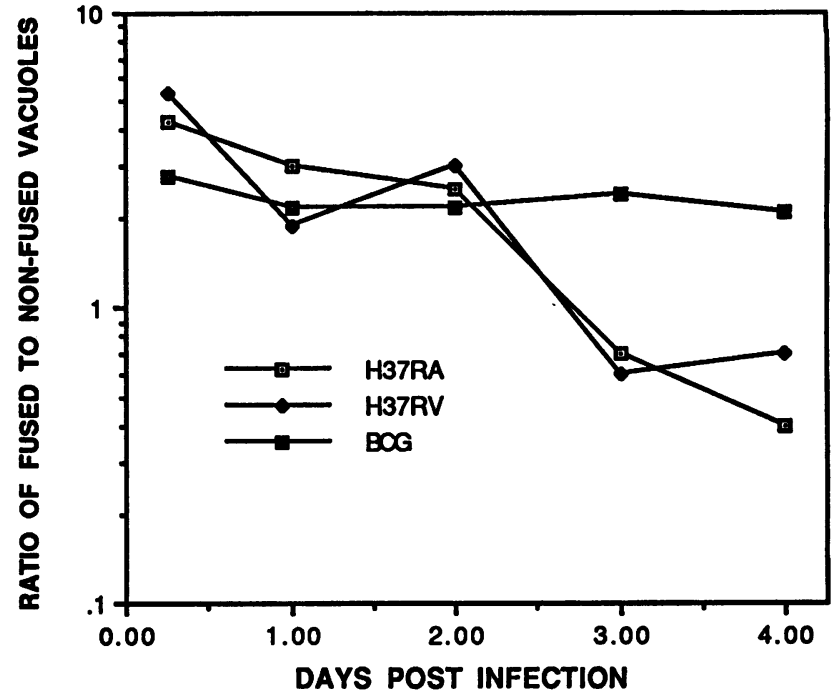

FIG. 4. Change of phagolysosome fusion patterns of viable H37Ra and H37Rv from predominantly fused to mostly nonfused over a 4-day period. The fusion profile did not change with BCG. The results shown are representative of three independent experiments.

enclosed within the layers of membrane. In contrast, the cytoplasm of H37Ra over time became increasingly filled with what appeared to be large lipid-containing vacuoles (Fig. 5d). The appearance of BCG bacilli changed little over the course of these experiments, although a very small number of these organisms was observed to take on either the H37Ra-like or H37Rv-like appearance.

The nature of the cellular vacuoles in which H37Ra and H37Rv resided also changed with time. Whereas at times shortly after infection most bacteria were found in vacuoles with clearly defined membranes and multiple organisms, this pattern slowly changed to one in which bacteria were increasingly found individually in vesicles with membranes tightly apposed to the bacterial cell surface, or apparently free in the cytoplasm, with no obvious membrane (Fig. 5d and e). Occasionally, individual tubercle bacilli appeared to be budding out of the larger vacuoles, as illustrated in Fig. 6 . In contrast, the vacuolar profile of heat-killed bacteria and of viable BCG changed little over time, and cell membranes were usually readily apparent (Fig. 5f).

It was possible that our inability to discern a membrane around some of these organisms was due to an artifact of microscopy. We therefore examined bacteria in crude cell lysates designed to wash away soluble cytoplasmic components while preserving some of the cellular architecture and leaving membrane components intact. At 4 days postinfection, infected monolayers were rinsed several times with PBS and then hypotonically lysed by the addition of ice-cold distilled water. Cells were then resuspended with the aid of a rubber policeman, pelleted, and resuspended in fixative containing tannic acid to further enhance membrane visualization. As a control, extracellular bacteria were grown under the same conditions as the infected monolayers but in the absence of J774.16 cells. As shown in Fig. 7, membranes were well preserved by this method, and bacteria can be clearly observed within vacuolar membranes. Two additional categories of bacteria were also identified by this method: those with a tightly adherent membrane and those 

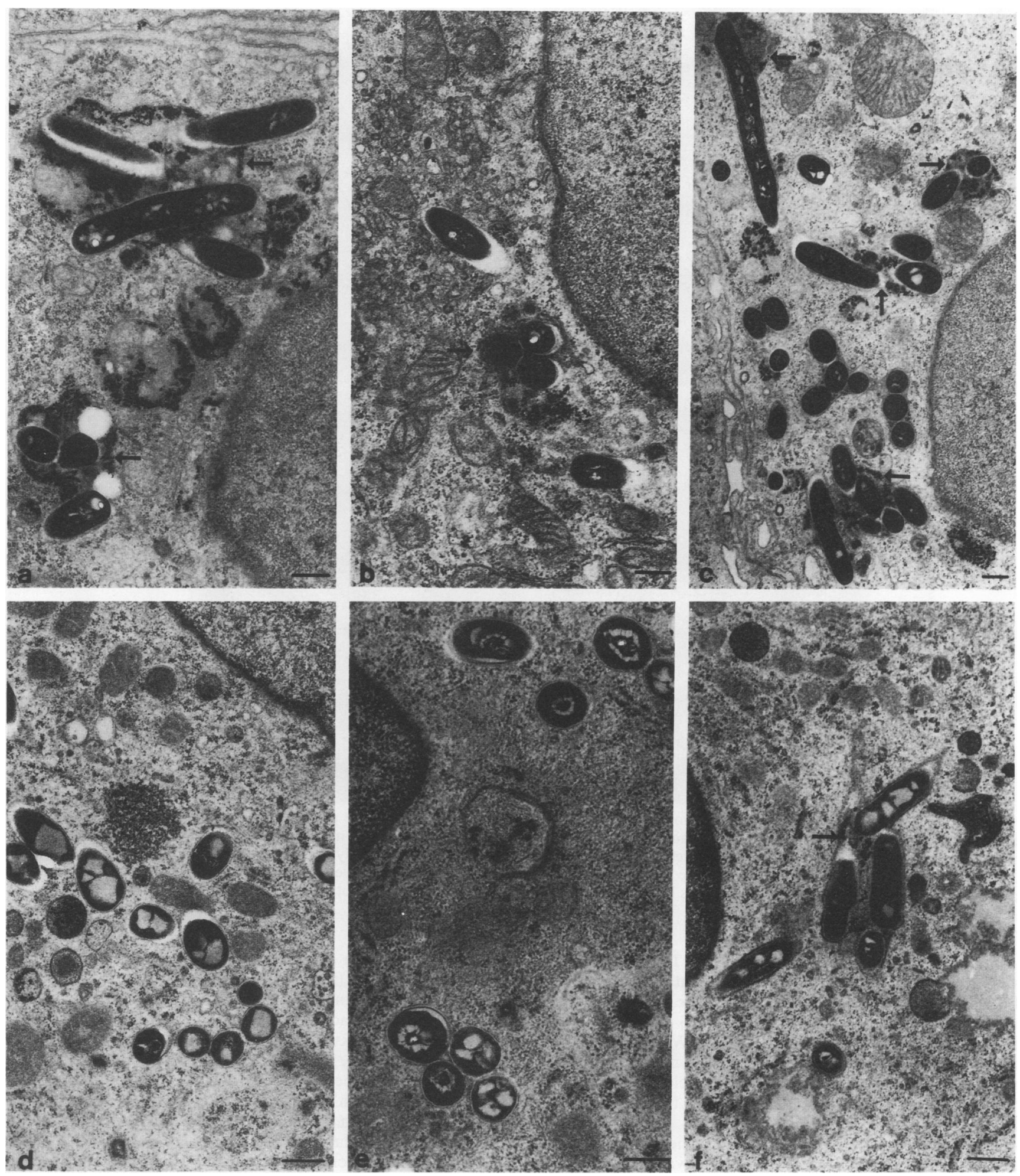

FIG. 5. Thoria-labelled J774.16 macrophages were infected with H37Ra (a), H37Rv (b), and BCG (c) for $4 \mathrm{~h}$ and H37Ra (d), H37Rv (e), and BCG (f) for 5 days. Arrows denote phagolysosome fusion. Note the phenotypic changes in bacteria after long-term infection in panels $\mathrm{d}$ and e. Bar, $0.5 \mu \mathrm{m}$. 


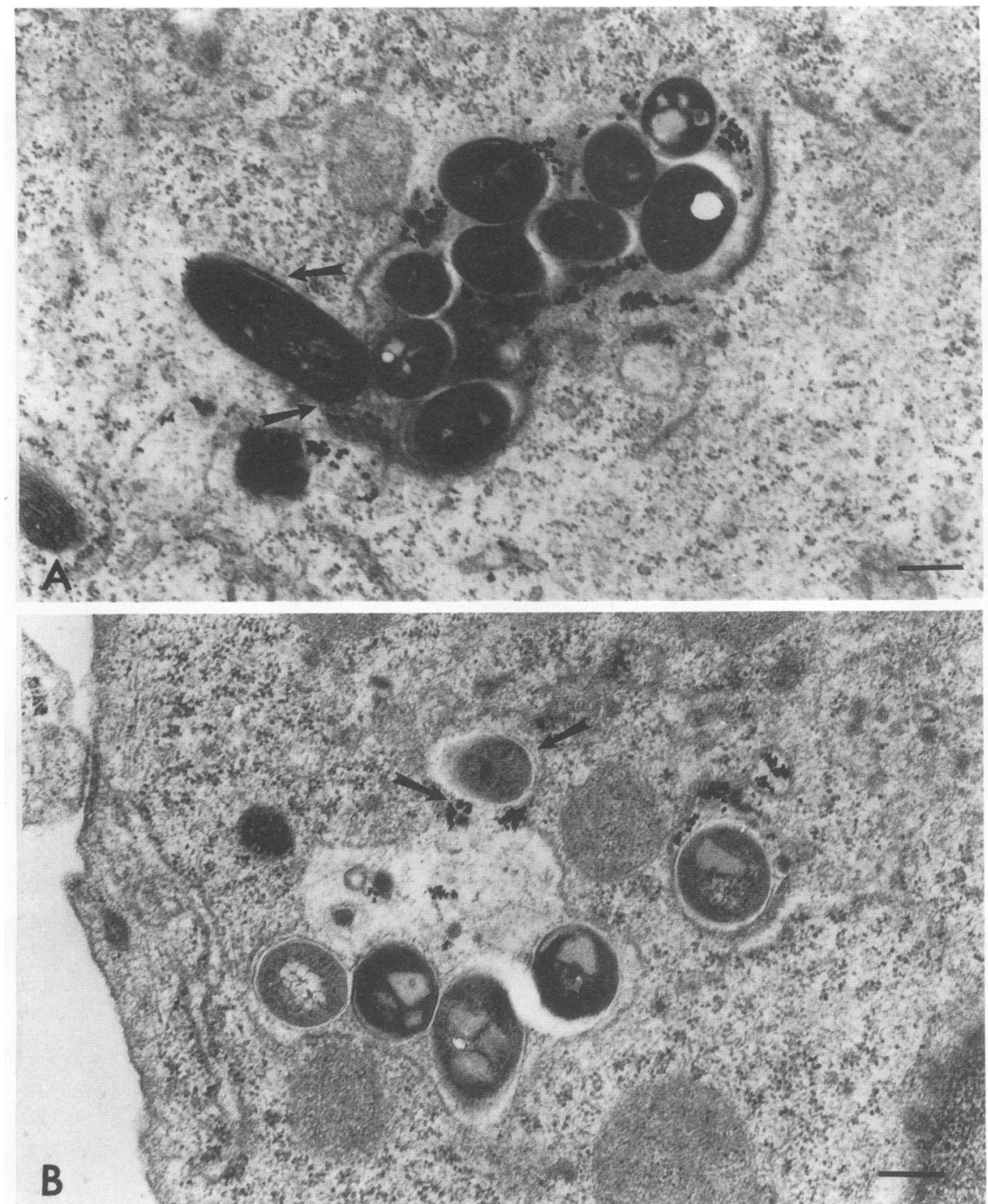

FIG. 6. Individual H37Ra bacilli appearing to bud out of a phagolysosome. Arrows denote portions of the vacuolar membrane that have become tightly adherent to the surface of the bacillus and the leading edge of the surrounding membrane that appears to be encircling bacilli prior to their being pinched off. Note the apparent exclusion of thoria particles from the newly forming phagosome in panel B. The bacillus in panel B appears to be in a later stage of the extrusion process than that in panel A; however, because these are still pictures and not sequential video images, temporal relationships between these images are inferred and cannot be determined with certainty. Bar, $0.4 \mu \mathrm{m}$.

with no apparent vacuolar membrane. These latter two categories are difficult to differentiate in intact cells because of the surrounding cytoplasm. Quantitative differences were observed among $\mathrm{H} 37 \mathrm{Rv}, \mathrm{H} 37 \mathrm{Ra}$, and BCG in the proportion of bacteria found in each type of vacuole. Whereas BCG was nearly always found surrounded by a distinct vacuolar membrane at 4 days, H37Rv was found in clearly membraneenclosed vacuoles only about half of the time, and H37Ra had an intermediate phenotype.

Bacterial multiplication and spread. The progression of bacterial infection was assayed in two ways (Fig. 8). By using an initial multiplicity of infection of approximately 10 bacteria added per macrophage, we assessed the degree of bacterial spread over a 4-day period on the basis of daily counts of the percentage of cells in the monolayer infected with bacteria. BCG infection did not spread appreciably during this time period, but the percentage of cells in the monolayer infected with $\mathrm{H} 37 \mathrm{Ra}$ increased from 30 to $50 \%$, and the proportion of those infected with $\mathrm{H} 37 \mathrm{Rv}$ tripled in the same period (Fig. 8a). Bacterial multiplication was inferred from the infectivity index, represented here as the total number of bacteria per 100 cells. After an initial lag period, only H37Rv bacteria appeared to multiply significantly over a 4-day period of observation (Fig. 8b).

To ascertain whether bacterial growth occurred equally well in fused and in nonfused vacuoles, the average number of bacteria present in each type of vacuole over a 7-day period was calculated (Fig. 9). The average number of H37Rv bacteria increased during the first 3 days postinfection, after which the number leveled off before dropping at 6 days (Fig. 9a). (This decrease more likely reflects a cycle of cell lysis and reinfection than a true reduction in the multiplication rate of the $\mathrm{H} 37 \mathrm{Rv}$ bacteria, as determined by viability counts and microscopical assays [19a].) In contrast, the average number of bacteria per fused vacuole did not increase over the 7-day period examined. No increases in 

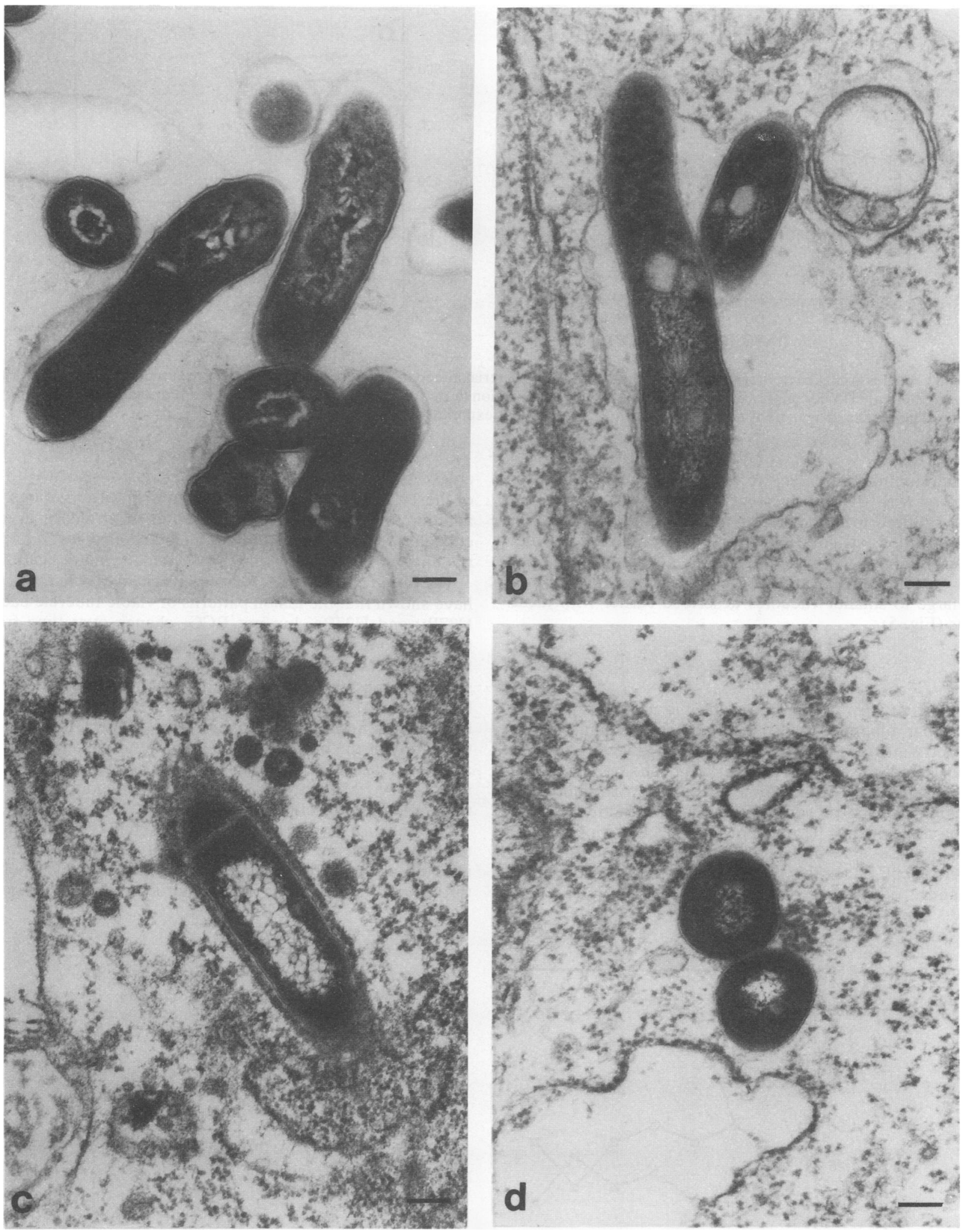

FIG. 7. J774.16 cells hypotonically lysed with ice-cold distilled water at 4 days postinfection to demonstrate the nature of the membranes surrounding the bacteria. (a) H37Rv grown extracellularly for 4 days under tissue culture conditions in cell culture medium supplemented with $20 \%$ fetal bovine serum (note that the bull's-eye phenotype becomes apparent during these conditions despite extracellular growth); (b) BCG bacteria clearly contained within a discrete membrane-bound vacuole; (c) H37Rv surrounded by a tightly apposed thickened membrane; (d) H37Rv bacteria free in cytoplasm with no discernible membrane around them. Bar, $0.2 \mu \mathrm{m}$. 

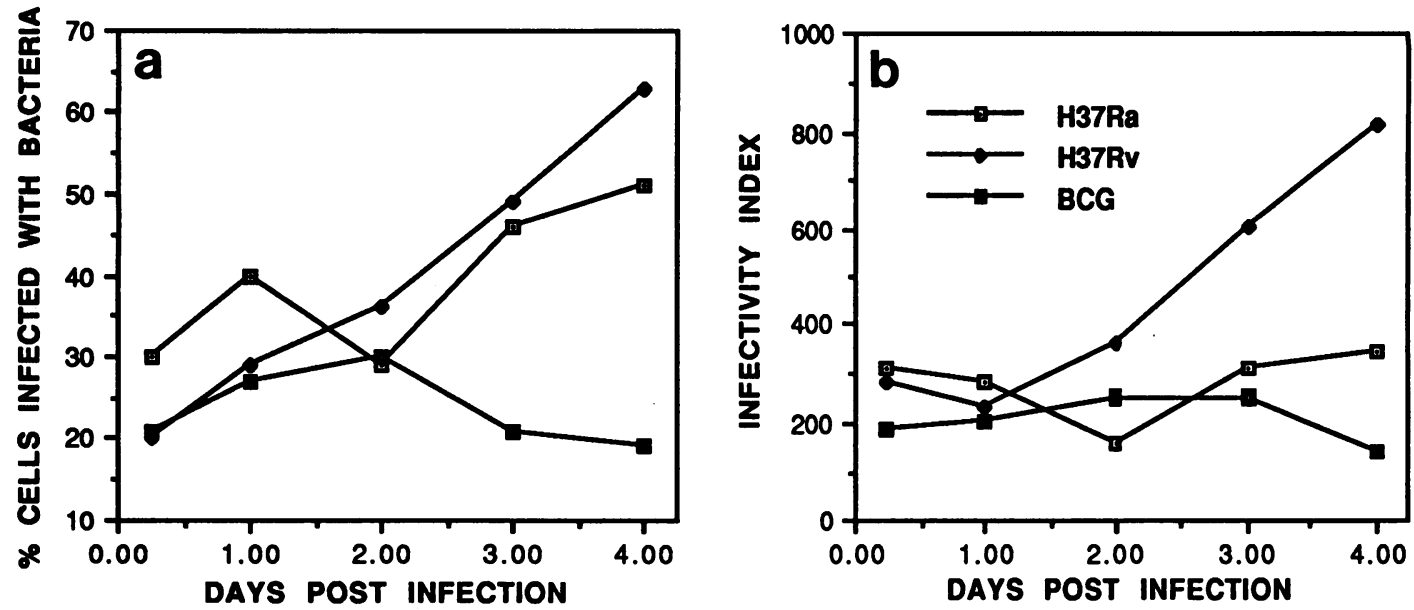

FIG. 8. Progression of bacterial infection over time. Spread of bacterial infection throughout the monolayer occurred with both $\mathrm{H} 37 \mathrm{Ra}$ and H37Rv (a), but only H37Rv underwent significant intracellular replication during a 4-day period (b). BCG neither replicated nor spread during this time. The results shown are representative of three independent experiments.

bacterial number were seen in either type of vacuole with H37Ra or with BCG, consistent with the lack of bacterial growth inferred from the infectivity indices reported in Fig. $8 \mathrm{~b}$. In addition, strains $\mathrm{H} 37 \mathrm{Rv}$ and H37Ra exhibited a net decrease after day 1 in the average number of bacteria per fused vacuole, consistent with the redistribution of bacteria from large to smaller vacuoles described earlier with these strains (Fig. 5).

\section{DISCUSSION}

Previous studies of $M$. tuberculosis in macrophages suggested that tubercle bacilli inhibit phagolysosome fusion upon entry into murine macrophages (1). However, this was demonstrated not to be a necessary condition for their survival, since $M$. tuberculosis opsonized with antibodies did undergo phagolysosome fusion but remained viable (2). In contrast, evidence has been presented suggesting that the fusion process may in fact be advantageous to infecting tubercle bacilli (5). In this report, we have proposed a novel mechanism of $M$. tuberculosis-phagolysosome interaction, an escape from fusion, that we believe is consistent with all of these prior (and seemingly incongruous) observations. In addition, several phenotypic correlates of virulence in mycobacteria have been identified, including changes in bacterial and vacuolar morphology and bacillary multiplication.

The fusion pattern of $M$. tuberculosis changes with time. We have shown that the fusion patterns of viable tubercle bacilli H37Rv and H37Ra, but not BCG, change over time from fused to nonfused vacuoles, but the mechanism responsible for this reversal is not clear. Four possibilities include (i) selective transfer of lysosomal components out of the phagolysosome, (ii) reentry of previously internalized bacteria through a non-fusion-related pathway, (iii) preferential multiplication of the bacterial population in nonfused vacuoles, and (iv) escape of bacilli from phagolysosomes to nonfusable vesicles. We favor the last interpretation but address each of the possibilities briefly below.

No evidence for the transfer of lysosomal contents out of the phagolysosome could be observed. Lysosomes in both infected and in uninfected cells remained heavily labelled throughout the course of these experiments as did phagoly-
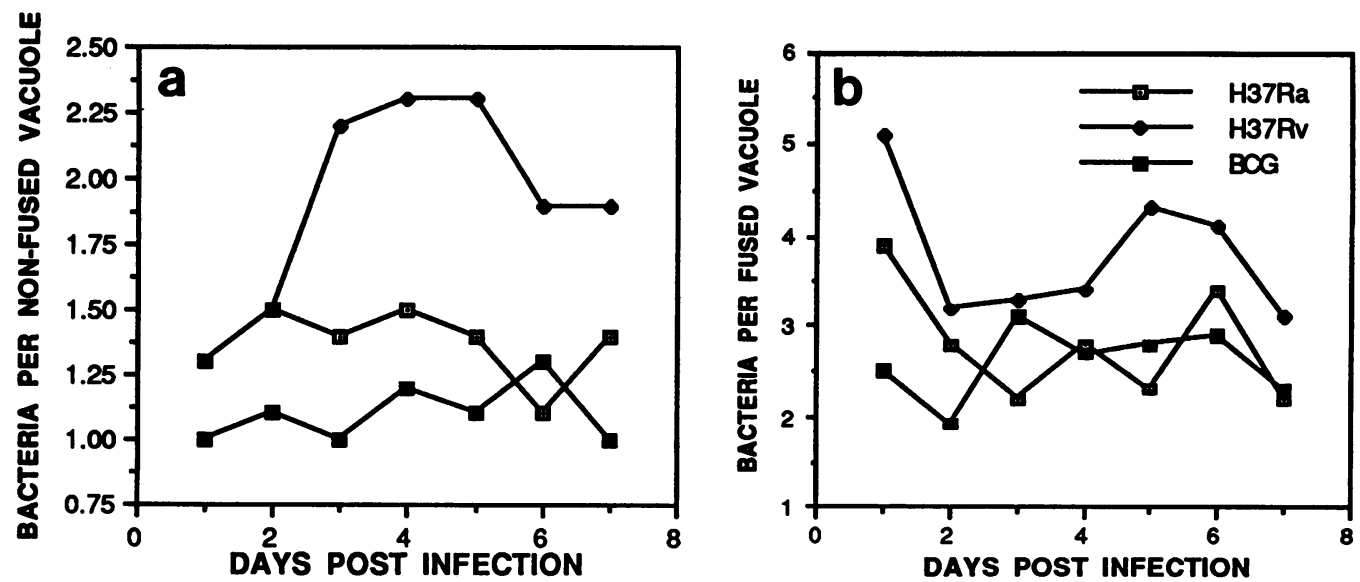

FIG. 9. Distribution of bacteria among vacuoles. The number of H37Rv bacteria increased in nonfused (a) but not in fused (b) vacuoles during a 7-day period. Little multiplication was seen in either type of vacuole with H37Ra and BCG. The results shown are representative of three independent experiments. 
sosomes containing all strains of dead bacteria and viable BCG bacilli.

Many facultative (and obligate) intracellular pathogens undergo genetic regulatory responses following uptake by host cells that facilitate their survival in the intracellular milieu (13). Little is known about the genetic response of $M$. tuberculosis to the intracellular environment of a macrophage. It is quite possible that intracellular tubercle bacilli express surface components that are not present on extracellularly grown bacilli and that these newly expressed ligands mediate uptake of these bacteria by novel pathways, including those that do not lead to phagolysosome fusion. We have found, by performing reinfection experiments with various serum conditions, that in the presence of heatinactivated serum the contribution of such effects is insufficient to explain the degree of fusion reversal we observed (19a).

Preferential multiplication of H37Rv occurred in nonfused vacuoles (Fig. 8), and it no doubt contributed to the fusion reversal pattern we observed. However, several lines of evidence indicate that bacterial multiplication in unfused vesicles alone is not sufficient to explain the decreasing fusion phenomenon we observed. Specifically, a similar pattern of fusion reversal was observed with H37Ra (Fig. 4) in the absence of significant increases assessed microscopically in either the number of bacteria per nonfused vacuole or total bacterial counts (Fig. 7). Morphological evidence suggests a net transfer of viable $M$. tuberculosis out of phagolysosomes that is not apparent with BCG or any strain of killed mycobacteria. This extrusion phenomenon becomes apparent by 1 day postinfection. Individual bacteria appear to bud out, pinching off membranes of the larger phagolysosomal vacuoles (Fig. 6). Early in this process, portions of the bacterial surface appear to fuse with contact points along the vacuolar membrane. These observations are consistent with the findings of Leake et al. (19) and Myrvik et al. (22), who previously described a similar membrane adhesion phenomenon that they believed correlated with bacterial virulence. At later times postinfection, the distribution of bacteria in the cell changes from one in which multiple bacilli reside in large vacuoles with clearly defined membranes to a picture of individual bacteria in tightly fitting vacuoles with difficult-to-discern, tightly apposed membranes (Fig. 5).

The nature of the membranes surrounding these bacteria is unclear but of significant interest to us. Recent studies indicate that a major histocompatibility complex (MHC) class I-mediated immune response, presumably governing development of cytotoxic $\mathrm{T}$ lymphocytes, is essential for controlling $M$. tuberculosis infection in mice $(10,14)$. MHC class I processing and presentation are thought to occur only for antigens in the cytoplasmic compartment (43), and these findings are difficult to explain if $M$. tuberculosis antigens reside only in a vacuolar compartment. One would have to argue, in the case of mycobacteria, that MHC class I antigen presentation could conceivably arise either from bacteria escaping directly from the tightly apposed membrane vesicles into the cytoplasm or from bacilli enclosed within a peculiar vesicular membrane that allowed export of mycobacterial antigens into the cytoplasm. While several investigators have failed to find tubercle bacilli free in the cytoplasm $(8,11)$, the appearance of the bacilli in our study is consistent with that reported by Myrvik et al. (22), who concluded that most $\mathrm{H} 37 \mathrm{Rv}$ bacteria at $24 \mathrm{~h}$ postinfection could be found free within the cytoplasm of rabbit alveolar macrophages. Demonstration of cytoplasmic localization of
M. tuberculosis would explain the MHC class I requirement for protection in the murine studies. We have provided suggestive electron microscopic evidence that some $M$. tuberculosis bacteria can escape from the tightly apposed membrane vesicle into the macrophage cytoplasm after several days (Fig. 7). However, without further analysis, one must exercise caution in distinguishing between a tubercle bacillus free in the cytoplasm and one which is encased in a tightly apposed vacuolar membrane, and investigations that will allow us to discriminate more precisely between these two possibilities are currently under way.

Among the wide variety of microbial mechanisms to resist intracellular killing, to our knowledge, no such strategy for escaping from a fused phagolysosome into another membrane-bound vesicle has been previously described. The mechanism by which this process occurs is not clear, but it is possible that bacterial surface components interact with ligands on the vacuole membrane which enable them to enter an existing recycling pathway. Alternatively, fusion of the lipid components on the bacterial surface with the lipid of the vacuolar membrane could conceivably create a tightly apposed vesicular membrane around the bacterium. While such a mechanism is unusual for a bacterium, many viruses depend upon fusion of their envelopes with the vacuolar membrane to establish infection of host cells $(40,41)$. The resistance of these secondary vesicles to fusion could occur in several ways, including budding of $M$. tuberculosis in such a fashion that a critical component of the membrane required for fusion is left in the phagolysosome, release of metabolic products by the bacterium that prevent the tight vesicle membrane from fusing, or dissolution of the surrounding membrane such that tubercle bacilli become free in the cytoplasm.

Bacterial degradation occurs slowly. Two disadvantages of earlier studies on the effect of phagolysosome fusion on bacterial viability in macrophages are that determinations of viability were often made after bacteria had resided in a phagolysosome for several days, and only half of the bacteria in the starting cultures were intact at the time of infection $(1,2)$. It was therefore difficult, in these earlier studies, to determine what percentage of the intact population was actually viable, and what percentage of the damaged population may have arisen because of the degradative effects of the phagolysosome.

The viability of intact mycobacteria is invariably difficult to assess with certainty, since the clumpiness of mycobacteria makes exact enumeration of viable organisms in any given culture by colony counts difficult. However, in our experiments, virtually all bacteria in our starting cultures were morphologically intact. Equally important, to reduce further the possibility that the viability of infecting organisms would be affected by long-term residence within a phagolysosome, we assayed phagolysosome fusion at very early times postinfection. The heat-killed bacterial cultures used similarly contained mostly bacteria which remained morphologically intact, allowing us to follow degradation of these bacteria over time. Degradation of mycobacteria in phagolysosomes was notably slow, a finding that has been reported before (2), and is consistent with the formidable characteristics of mycobacterial cell walls. This resistance to degradation could also explain prolonged immunological response to mycobacterial antigens after infection.

Additional correlates of virulence: bacterial multiplication and morphology. Several additional correlates of virulence were noted during the course of these studies. In particular, rates of intracellular multiplication differed among the 
strains tested, with the virulent strain H37Rv having significantly higher levels of multiplication than either of the two attenuated strains. This observation is consistent with the findings of Shepard, who first provided evidence that the ability to multiply intracellularly in a variety of cell lines correlated with virulence $(32,33)$.

In addition, significant changes in both bacterial morphology and vacuolar morphology were observed over time in cells infected with H37Rv and H37Ra. Less obvious differences were seen with BCG. The bull's-eye phenotype of H37Rv bacteria resembles that seen with chlamydiae (23) and appears to reflect condensed nucleic acid. It is possible that this phenotype represents a genetic regulatory response of tubercle bacilli to the intracellular environment as it does for chlamydiae $(3,15)$. We noted that similar changes did not occur with either H37Ra or BCG bacteria, and it is interesting to speculate that the decreased virulence of these attenuated strains is due to regulatory mutations that render them unable to respond fully to the intracellular environment.

Differences between H37Ra and BCG were also observed with respect to the morphology of the intracellular bacteria. Whereas the appearance of BCG bacteria did not change over time, H37Ra bacteria developed what appeared to be large lipid inclusion vacuoles (Fig. 5d). This suggests that H37Ra may undergo a first-phase response to the intracellular environment and that BCG cannot respond in a similar fashion. Another intriguing possibility is that H37Ra increases lipid synthesis after uptake but is blocked in its transport out of the bacterial cell. The fusion lag we observed upon entry of H37Ra bacteria into macrophages is puzzling but reflects an additional defect in the regulatory function of H37Ra.

In conclusion, our findings show that most tubercle bacilli were directed to phagolysosomes immediately after engulfment by macrophages. Individual $\mathrm{H} 37 \mathrm{Rv}$ and $\mathrm{H} 37 \mathrm{Ra}$ bacteria later appeared to bud out from the fused phagolysosomes into vacuoles whose membranes were tightly apposed to the bacterial surface and which did not fuse with secondary lysosomes. Eventually, the fusion pattern changed from one in which H37Rv bacteria resided in predominantly fused phagolysosomes to one of mostly nonfusion. During the same time period, the number of intracellular H37Rv bacteria increased more than 10-fold. Avirulent BCG bacteria did not exhibit this escape-from-fusion phenomenon nor did they undergo significant bacterial multiplication during the course of these experiments. These findings raise the intriguing possibility that temporary residence within a phagolysosome stimulates a response of $M$. tuberculosis to the intracellular environment that facilitates its long-term survival and replication. We anticipate that further study of the differences between these virulent and avirulent strains will provide insights into the molecular mechanisms by which the tubercle bacillus senses and responds to its intracellular environment.

\section{ACKNOWLEDGMENTS}

We thank J. Flynn, C. Brosnan, and W. Jacobs for critical reading of the manuscript.

This work was supported partially by National Institutes of Health grant AI07118 and a Howard Hughes Medical Institute fellowship (K.A.M.)

\section{REFERENCES}

1. Armstrong, J. A., and P. D. Hart. 1971. Response of cultured macrophages to Mycobacterium tuberculosis, with observations on fusion of lysosomes with phagosomes. J. Exp. Med. 134:713 740.
2. Armstrong, J. A., and P. D. Hart. 1975. Phagosome-lysosome interactions in cultured macrophages infected with virulent tubercle bacilli. Reversal of the usual nonfusion pattern and observations on bacterial survival. J. Exp. Med. 142:1-16.

3. Barry, C. E., III, S. F. Hayes, and T. Hackstadt. 1992. Nucleoid condensation in Escherichia coli that express a chlamydial histone homolog. Science 256:377-379.

4. Bloom, B. R., and C. J. L. Murray. 1992. Tuberculosis: commentary on a reemergent killer. Science 257:1055-1064.

5. Brown, C. A., P. Draper, and P. D. Hart. 1969. Mycobacteria and lysosomes: a paradox. Nature (London) 221:658-660.

6. Canetti, G. 1955 . The tubercle bacillus in the pulmonary lesion of man. Springer, New York.

7. Canetti, G. 1965. Present aspects of bacterial resistance in tuberculosis. Am. Rev. Respir. Dis. 92:687-703.

8. Crowle, A. J. 1986. Studies of antituberculosis chemotherapy with an in vitro model of human tuberculosis. Semin. Respir. Infect. 1:262-264.

9. Damiani, G., C. Kiyotaki, W. Soeller, M. Sasada, J. Peisach, and B. R. Bloom. 1980. Macrophage variants in oxygen metabolism. J. Exp. Med. 152:808-822.

10. DeLibero, G., I. Flesch, and S. H. E. Kaufmann. 1988. Mycobacteria-reactive Lyt-2 + T cell lines. Eur. J. Immunol. 18:5966.

11. Douvas, G. S., E. M. Berger, J. E. Repine, and A. J. Crowle. 1986. Natural mycobacteriostatic activity in human monocytederived adherent cells. Am. Rev. Respir. Dis. 134:44-48.

12. Fields, P. I., E. A. Groisman, and F. Heffron. 1989. A Salmonella locus that controls resistance to microbicidal proteins from phagocytic cells. Science 243:1059-1062.

13. Finlay, B. B., and S. Falkow. 1989. Common themes in microbial pathogenicity. Microbiol. Rev. 53:210-230.

14. Flynn, J. L., M. M. Goldstein, K. J. Triebold, B. Koller, and B. R. Bloom. 1992. Major histocompatibility complex class I-restricted T cells are required for resistance to Mycobacterium tuberculosis infection. Proc. Natl. Acad. Sci. USA 89:1201312017.

15. Hackstadt, T., W. Baehr, and Y. Ying. 1991. Chlamydia trachomatis developmentally regulated protein is homologous to eukaryotic histone H1. Proc. Natl. Acad. Sci. USA 88:39373941.

16. Hart, P. D., J. A. Armstrong, C. A. Brown, and P. Draper. 1972. Ultrastructural study of the behavior of macrophages toward parasitic mycobacteria. Infect. Immun. 5:803-807.

17. Joiner, K. A., S. A. Fuhrman, H. M. Miettinen, L. H. Kasper, and I. Mellman. 1990. Toxoplasma gondii: fusion competence of parasitophorous vacuoles in Fc receptor-transfected fibroblasts. Science 249:641-646.

18. Kim, K., I. H. Kim, K. Y. Lee, S. G. Rhee, and E. R. Stadtman. 1988. The isolation and purification of a specific "protector" protein which inhibits enzyme inactivation by a thiol/Fe(III)/O2 mixed-function oxidation system. J. Biol. Chem. 263:4704 4711.

19. Leake, E. S., J. R. Ockers, and Q. N. Myrvik. 1977. In vitro interactions of the BCG and Ravenel strains of Mycobacterium bovis with rabbit macrophages: adherence of the phagosomal membrane to the bacterial cell wall and the problem of the peribacillary space. J. Reticuloendothel. Soc. 22:129-147.

19a.McDonough, K. A., Y. Kress, and B. R. Bloom. Unpublished observations.

20. Moulder, J. W. 1985. Comparative biology of intracellular parasitism. Microbiol. Rev. 49:298-337.

21. Murray, C. J., K. Styblo, and A. Rouillon. 1990. Tuberculosis in developing countries: burden, intervention and cost. Bull. Int. Union Tuberc. 65:6-24.

22. Myrvik, Q. N., E. S. Leake, and M. J. Wright. 1984. Disruption of phagosomal membranes of normal alveolar macrophages by the H37Rv strain of Mycobacterium tuberculosis. A correlate of virulence. Am. Rev. Respir. Dis. 129:322-328.

23. Pearce, J. H. 1986. Early events in chlamydial infection. Ann. Inst. Pasteur Microbiol. 137:325-332.

24. Portnoy, D. A., P. S. Jacks, and D. J. Hinrichs. 1988. Role of hemolysin for the intracellular growth of Listeria monocytoge- 
nes. J. Exp. Med. 167:1459-1471.

25. Rich, A. R. 1951. The pathogenesis of tuberculosis, 2nd ed. Charles C Thomas, Publisher, Springfield, Ill.

26. Riley, R. L., C. C. Mills, W. Nyka, N. Weinstock, P. B. Storey, L. U. Sultan, M. C. Riley, and W. F. Wells. 1959. Aerial dissemination of pulmonary tuberculosis. Am. J. Hyg. 70:185196.

27. Riley, R. L., and F. O'Grady. 1961. Airborne contagion. Macmillan Publishing Co., Inc., New York.

28. Rook, G. A. W., J. Steele, M. Ainsworth, and B. R. Champion. 1986. Activation of macrophages to inhibit proliferation of Mycobacterium tuberculosis: comparison of the effects of gamma-interferon on human monocytes and murine peritoneal macrophages. Immunology 50:333-338.

29. Sansonetti, P. J., A. Ryter, P. Clerc, A. T. Maurelli, and J. Mounier. 1986. Multiplication of Shigella flexneri within HeLa cells: lysis of the phagocytic vacuole and plasmid-mediated contact hemolysis. Infect. Immun. 51:461-469.

30. Schlesinger, L. S., C. G. Bellinger-Kawahara, N. R. Payne, and M. A. Horwitz. 1990. Phagocytosis of Mycobacterium tuberculosis is mediated by human monocyte complement receptors and complement component C3. J. Immunol. 144:2771-2780.

31. Schmid, S. L., R. Fuchs, P. Male, and I. Mellman. 1988. Two distinct subpopulations of endosomes involved in membrane recycling and transport to lysosomes. Cell 52:73-83.

32. Shepard, C. C. 1956 . Growth characteristics of tubercle bacilli and certain other mycobacteria in HeLa cells. J. Exp. Med. 105:39-55.

33. Shepard, C. C. 1958. A comparison of the growth of selected mycobacteria in HeLa, monkey kidney and human amnion cells in tissue culture. J. Exp. Med. 107:237-250.
34. Sibille, Y., and H. Y. Reynolds. 1990. Macrophages and polymorphonuclear neutrophils in lung defense and injury. Am. Rev. Respir. Dis. 141:471-501.

35. Sibley, L. D., J. L. Krahenbuhl, G. M. W. Adams, and E. Weidner. 1986. Toxoplasma modifies phagosomes by secretion of a vesicular network rich in surface proteins. J. Cell Biol. 103:867-874.

36. Sibley, L. D., E. Weidner, and J. L. Krahenbuhl. 1985. Phagosome acidification blocked by intracellular Toxoplasma gondii. Nature (London) 315:416-419.

37. Straley, S. C., and P. A. Harmon. 1984. Yersinia pestis grows within phagolysosomes in mouse peritoneal macrophages. Infect. Immun. 45:655-659.

38. Verhoef, J. 1991. Host-pathogen relationships in respiratory tract infections. Clin. Ther. 13:172-180.

39. Wells, W. F. 1955. Airborne contagion and air hygiene. Harvard University Press, Cambridge, Mass.

40. White, J., M. Kielian, and A. Helenius. 1983. Membrane fusion of enveloped animal viruses. Q. Rev. Biophys. 16:151-195.

41. Wilschut, J., and D. Hoekstra. 1991. Membrane fusion. Marcel Dekker, Inc., New York.

42. Wilson, C. B., V. Tsai, and J. S. Remington. 1980. Failure to trigger the oxidative burst of normal macrophages. Possible mechanism for survival of intracellular pathogens. J. Exp. Med. 151:328-346.

43. Yewdell, J. W., and J. R. Bennink. 1990. The binary logic of antigen processing and presentation to $T$ cells. Cell 62:203-206.

44. Zeichner, S. L. 1983. Isolation and characterization of macrophage phagosomes containing infectious and heat-inactivated Chlamydia psittaci: two phagosomes with different intracellular behaviors. Infect. Immun. 40:956-966. 